\title{
Sarcomas Associated with Metal Implants in Orthopedic Surgery and Traumatology. A Report of 3 Cases in 2 Patients
}

\author{
Israel Rubio-Saez ${ }^{1 *}$, Luis Rodrigo Merino-Rueda ${ }^{2}$, Javier Alonso-Sanz ${ }^{1}$, Carmen Iglesias-Urraca ${ }^{1}$, Manuel \\ Peleteiro-Pensado ${ }^{1}$, Jose Juan Pozo-Kreilinger ${ }^{1}$ and Eduardo Jose Ortiz-Cruz ${ }^{1}$ \\ ${ }^{1}$ Hospital Universitario La Paz, Madrid, Spain \\ ${ }^{2}$ Hospital Universitario 12 De Octubre, Madrid, Spain

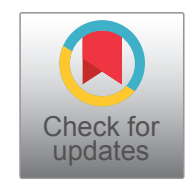

\begin{abstract}
We presented 3 cases of sarcomas associated with metal implants in 2 patients around metal implants with a previous history of chronic inflammation due to initial high energy trauma, several surgical operations, and implants in the region. Sarcoma rising in association with a metallic orthopedic prosthesis or hardware is an infrequent, but well documented complication. It is important to suspect its presence so that avoiding diagnostic errors that delay treatment and compromise the prognosis.
\end{abstract}

\section{Keywords}

Sarcoma, Metal, Implants, Fibrosarcoma, MPSNT

\section{Introduction}

The use of metal implants in Orthopedics is constant for the treatment of degenerative pathology as well as fractures [1]. Complications associated with metal implants are common, with infection being the most common [2,3]. There are other less common, but potentially very serious; within this group the cytogenetic toxicity associated with metal [4]. In addition, traumatic injury has also been suggested as a possible etiological factor in sarcoma development $[5,6]$ despite no established causal relationship $[7,8]$. The sarcomagenesis around metal implants is a rare but possible complication, the current experience is based on case reports and small series [1,9-12]. Clinical presentation is usually atypical which can result in delays and diagnostic errors. This work presents 3 cases of sarcomas associated with metal implants in 2 patients in a single center and their possible predisposing factors. Patients gave their informed consent for publication.

\section{Case-1}

A 58-year-old healthy male, who in February 2011 suffered motor vehicle accident with diagnosis of open fracture of the forearm Gustilo type IIIB associated with brachial plexus injury (BPI). After initial hemodynamic stabilization, emergent surgery was carried out for debridement and external fixation. 14-days later, he underwent open reduction and internal fixation of the open fracture of the forearm by radius and ulnar plating, soft tissue reconstruction with free flap of contralateral latissimus dorsi by the Plastic Surgery Service (Figure 1a).
During 2012 and 2013, due to the persistence of BPI, he required other procedures (nerve graft to median nerve, re-anchorage of latissimus dorsi tendon, humeral bone exostosis extirpation and latissimus dorsi tendon transfer to brachial biceps) making a sum of six different surgical operations in his arm for sequelae treatment.

In July 2012, a Total Knee Arthroplasty (TKA) was performed because of severe knee osteoarthritis. Since the beginning, he presented poor evolution, with pain and functional limitation; finally, two years later, in May 2014, he was diagnosed with aseptic loosening, so he underwent one-stage left TKA revision (Figure 1a). In March 2017, the patient started with a painful fast-growing mass in the left forearm, reaching about $10 \mathrm{~cm}$ in 3-months. The mass was radiologically evaluated (Figure 1b). Percutaneous biopsy was performed, showed a malignant spindle cell proliferation without any immunohistochemically cell differentiation, revealing a suspicious diagnosis of fibrosarcoma of low- intermediate grade

*Corresponding author: Rubio Sáez Israel, MD, Orthopaedic Surgeon Consultant, Hospital Universitario La Paz, Madrid, Spain

Accepted: November 10, 2020

Published online: November 12, 2020

Citation: Rubio-Saez I, Merino-Rueda LR, Alonso-Sanz J, et al. (2020) Sarcomas Associated with Metal Implants in Orthopedic Surgery and Traumatology. A Report of 3 Cases in 2 Patients. J Orthop Surg Tech 3(2):194-198 
Citation: Rubio-Saez I, Merino-Rueda LR, Alonso-Sanz J, et al. (2020) Sarcomas Associated with Metal Implants in Orthopedic Surgery and Traumatology. A Report of 3 Cases in 2 Patients. J Orthop Surg Tech 3(2):194-198
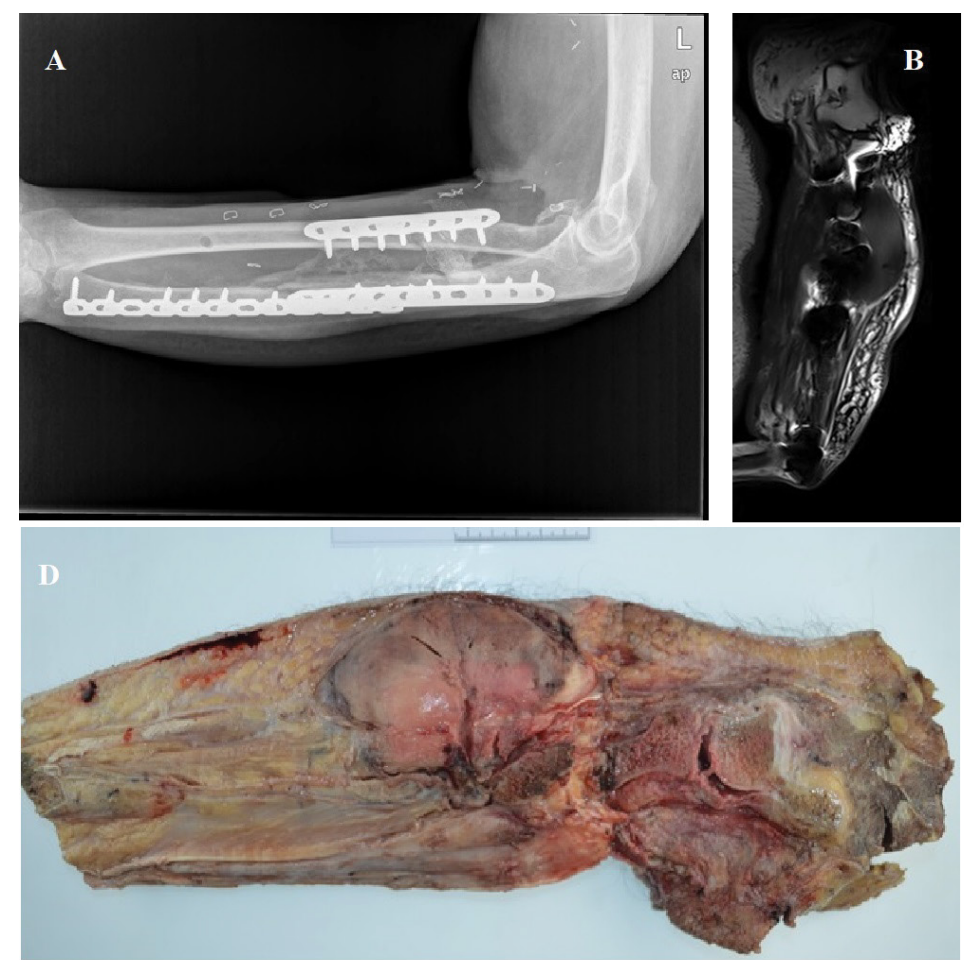

Figure 1: Case 1 (Elbow) A) Lateral radiograph that depicts a fracture of the forearm synthesized by double plating; B) Sagittal T1-enhanced MRI with tumor around osteosynthesis material in proximal forearm third; C, D) Pathological anatomy pieces showing contact between the tumor and the metal material; E) Histologically images that show a dense malignant mesenchymal spindle cell tumor with a classical herringbone pattern and with no other morphological or immunohistochemical differentiation compatible with high grade fibrosarcoma.

[13]. After staging work-up in which the presence of metastasis was ruled out, it was decided to perform local surgical treatment. Due its extra-compartmental location, poor condition of soft parts and sequelae of neurological injury, it was decided to perform left above-elbow amputation (Figure 1c and Figure 1d). Histologically analysis showed a dense malignant mesenchymal spindle cell tumor with a classical herringbone pattern and with no other morphological or immunohistochemical differentiation. A diagnosis of conventional fibrosarcoma of soft tissue, grade 2, was made (Figure 1e). From 2017 to 2019, the patient was assessed every 3-months by upper left limb Magnetic Resonance Imaging (MRI) and chest computed tomography (CT) without any suggestive images of local relapse or pulmonary metastases. In February 2019, he began with knee effusion and limited left knee range of motion. On simple radiograph, it had no pathological findings (Figure 2a). Synovial fluid of inflammatory characteristics was obtained by arthrocentesis ( 2250 cells, 44\% neutrophils, glucose $12 \mathrm{mg} / \mathrm{dl}$, proteins $4.1 \mathrm{~g} / \mathrm{dl}$ ) and the microbiological study ruled out infection. With the diagnosis of synovitis was carried out an open synovectomy of the left knee (Figure 2b), detecting an intraarticular mass of nonspecific characteristics that was studied histologically with diagnosis of fibrosarcoma of soft tissues, grade 3. Post-surgical MRI illustrated a persistent periprosthetic tumoral mass (Figure 2c). Again, the staging study discarded metastases. The limb-sparing surgery was contraindicated, due to great tumor contamination of the previous surgery. Therefore, an above knee amputation was indicated (Figure 2d) and no adjuvant therapies were indicated. After 3-months, the patient suffered an episode of ventricular tachycardia in the context of known ischemic heart disease and died.

\section{Case-2}

A 29-year-old woman suffered a severe gunshot wound in her left forearm in July 2005 and she was diagnosed of Gustilo type IIIC open fracture of proximal forearm. According to the medical reports provided, she had significant loss of bone substance at the level of the ulna, as well as soft tissue coverage. She had a surgical debridement and reconstruction with a vascularized fibula flap and plating, vascular bypass from brachial artery to radial artery, sural nerve graft for ulnar nerve reconstruction and coverage by latissimus dorsi free flap. Despite the initial debridement, abundant traces of shrapnel remained (Figure $3 a$ and Figure $3 b$ ). Consequently, her elbow persisted stiff, but with a competent hand.

In June 2019, 14 years after the initial injury, she went to our clinic complaining of a painless mass in the left forearm on the anterior side of the elbow. MRI and CT showed a $10 \times$ $4 \mathrm{~cm}$ soft tissue mass in the flexor origin of the elbow encompassing the metal remains (Figure 3c). Percutaneous biopsy was done and was compatible with high-grade fibrosarcoma with $\mathrm{Ki}-67$ proliferation index of $35 \%$. Extension work-up was negative for metastases After evaluation in Sarcomas Committee a surgical treatment was decided, and limb-sparing surgery was contraindicated and an above-elbow amputation with wide margins was done (Figure $3 \mathrm{~d}$ and Figure $3 e$ ). 
Citation: Rubio-Saez I, Merino-Rueda LR, Alonso-Sanz J, et al. (2020) Sarcomas Associated with Metal Implants in Orthopedic Surgery and Traumatology. A Report of 3 Cases in 2 Patients. J Orthop Surg Tech 3(2):194-198
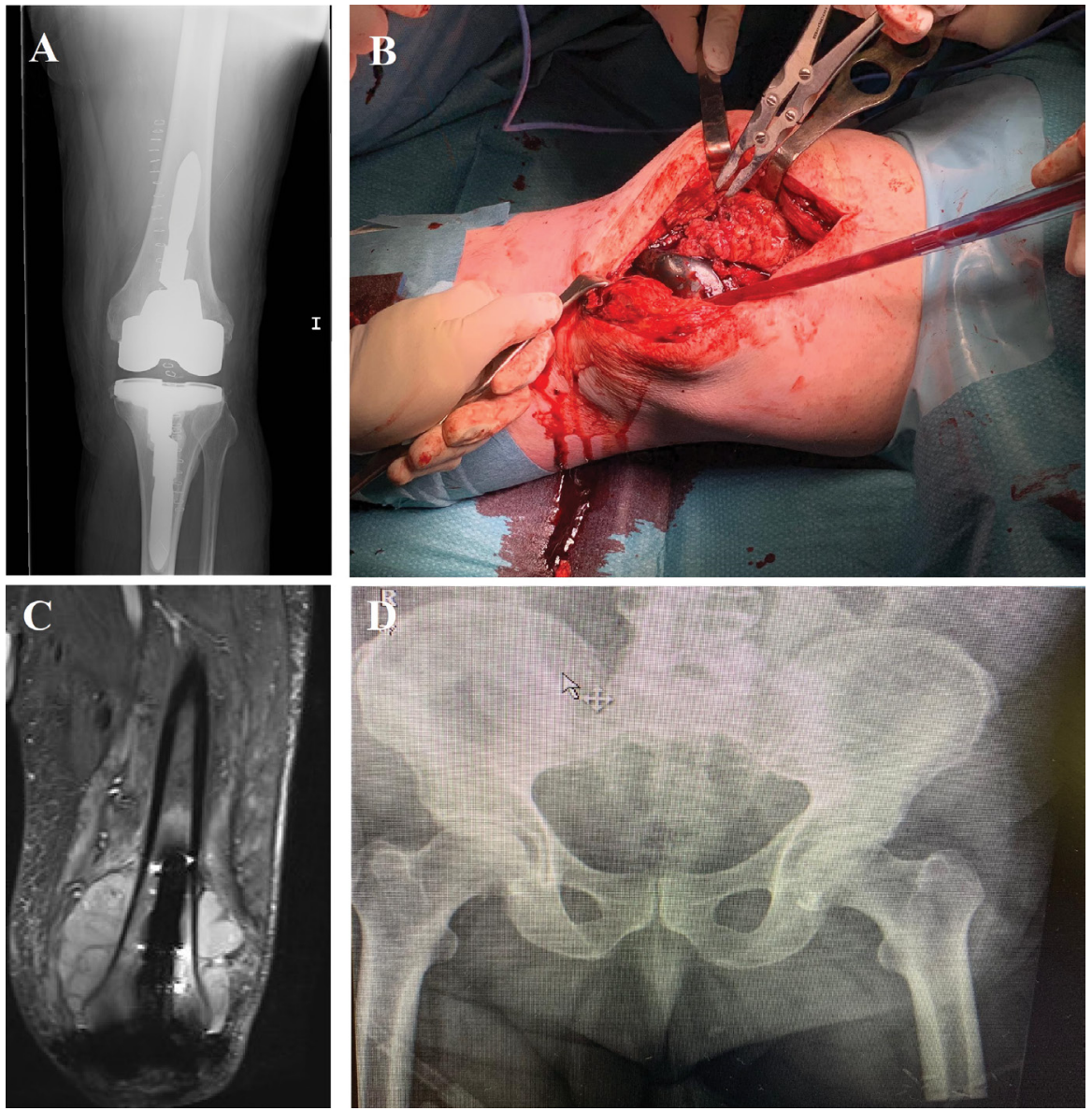

Figure 2: Case 1 (Knee) A) Anteroposterior radiograph showing left knee revision prosthesis; B) Intraoperative picture depicting the mass in open synovectomy; C) Coronal T2-enhanced MRI showing the tumor around left knee replacement prostheses; D) Anteroposterior radiograph showing proximal third left femur amputation.
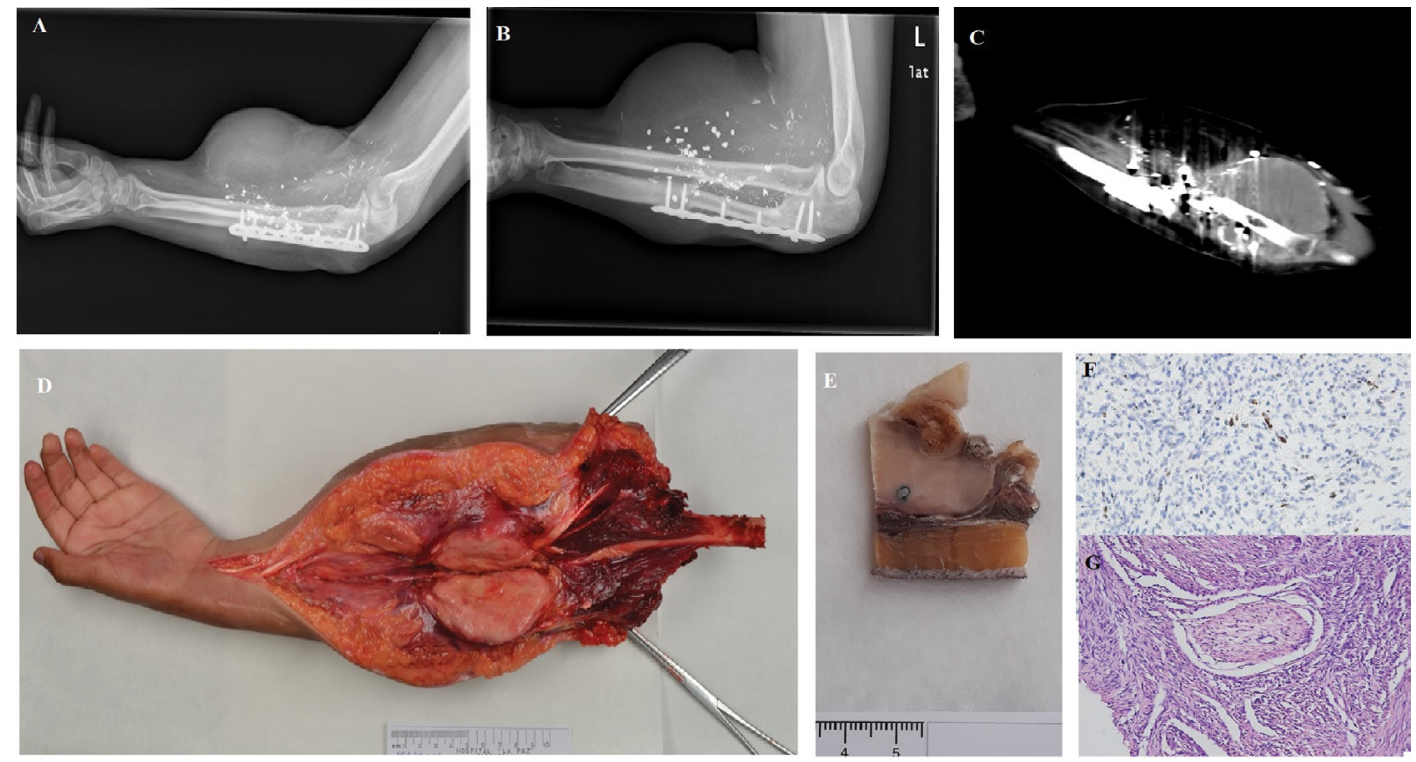

Figure 3: Case $2 \mathrm{~A}, \mathrm{~B}$ ) Lateral radiographs show ulna fracture reconstructed by latissimus dorsi free flap and plating and posterior free fibular flap for ulna non-union; C) CT with a tumor surrounding forearm proximal third osteosynthesis and shrapnel; D) Pathological anatomy pieces showing a big mass in flexor compartment of the forearm; $E$ ) and metallic shrapnel inside the tumor; $(F, G)$ Histological images that represent an extensive loss of expression of H3K27me3 in the tumor cellularity, guiding the final diagnosis towards a high grade malignant peripheral nerve sheath tumor (MPNST). 
Citation: Rubio-Saez I, Merino-Rueda LR, Alonso-Sanz J, et al. (2020) Sarcomas Associated with Metal Implants in Orthopedic Surgery and Traumatology. A Report of 3 Cases in 2 Patients. J Orthop Surg Tech 3(2):194-198

Interestingly the analyzed tumor of the amputation, showed in immunohistochemistry patchy positivity against CD56, CCD10, isolated CD57 positive cells, and an extensive loss of expression of $\mathrm{H} 3 \mathrm{~K} 27 \mathrm{me} 3$ in the tumor cellularity, guiding the final diagnosis towards a high grade malignant peripheral nerve sheath tumor (MPNST) (Figure $3 \mathrm{f}$ and Figure $3 \mathrm{~g}$ ). She received adjuvant chemotherapy (Epirrubicin/ Ifosfamide). Currently, twelve months later, the patient is disease-free and in process of prosthetic fitting.

\section{Discussion}

The presentation of a malignant tumor associated with a metal implant is a serious and uncommon complication. From 1950 to 2001, only 31 cases of sarcomas associated with metal implants [1] had been reported. Up to date, the longest English-speaking published review was done by Visuri, et al. [9] that consisted of 46 malignant tumors associated with Total Hip Arthroplasties (THA). Analyzing the main series so far, the undifferentiated pleomorphic sarcoma (UPS), previously designated as malignant fibrous histiocytoma, was the most common histology type [1,3], followed by osteosarcoma $[1,3]$, and then other sorts of tumors such as angiosarcomas $[4,12,13]$, usually of aggressive high or intermediate grade $[1,9,10]$. The main series state of an average time of appearance of neoplastic lesions ranging from 6 -years on average [1] to 18.8-years [10] and the outcomes were devastating $[1,9,10]$, tumors with aggressive behavior, reaching mortality of up to $77 \%$ per year [9].

Regarding possible risk factors involved in carcinogenesis, there is no consensus among the different authors. Most of the detected cases are related to THA $[1,9,10]$. However, there are patients affected with a single screw in the hip [11], a plate for osteosynthesis in femur [1], tibia [12,14], humerus $[15]$ or TKA $[16,17]$. Therefore, there is no clear relationship between the amount of metal and the carcinogenic potential. On the other hand, although THA is the most frequently implant associated $[1,9,10]$ according to the Finnish record [18] there is a lower incidence of soft tissue sarcomas (STS) in THA patients than the general population, but a higher incidence of STS has been observed in patients with a metal-on-metal hip prosthesis, with respect to the general population and wearers of non-metal-on-metal prostheses.

There are few published metal implant-associated cases of fibrosarcoma [5] and MPNST [1] such as two of our series. In veterinary researches, there are more reported cases in relation to microchips [19-22], due to the abnormal inflammatory response per foreign body. Carcinogenesis associated with metal implants has been studied in animal models [4]. Subcutaneous biomaterial devices were implanted in rats observing at 2-years of monitoring the appearance of malignant tumors in up to $25 \%$ of cases, again UPS being the most common. Some authors theorize on the formation of a capsule around the metal that would induce a proliferative reaction inducing cellular atypia as a preneoplastic substrate $[19,20]$.

On the other hand, initial high energy trauma or iatrogenic radiation may play an important role in sarcomagenesis [58]. Chronic inflammation has already been linked to sarcoma appearance [23-29]. Activation of inflammatory cell signaling pathways could be responsible for protooncogene activation and resulting in neoplastic cells. This could be related to the average time of occurrence of these lesions, which is usually long, probably so that these physiological changes can occur.

\section{Conclusion}

We describe these two exceptional cases of STS associated with metal implants. In the first patient, it depicted the appearance of the same type of tumor (Fibrosarcoma grade 3) on two different implants, which could speak in favor of an individual predisposition to suffer this type of lesions not yet clearly determined or an extremely rare case of distant metastasis. The second case, that lead to a MTPNS, is exceptional and only one case has been reported [1]. As described in our cases, the presentation is not pathognomonic and late diagnosis can be frequent.

\section{References}

1. Keel S, Jaffe K, Nielsen, et al. (2001) Orthopaedic implant-related sarcoma: A study of twelve cases. Modern Pathology 14: 969977.

2. Pulido L, Ghanem E, Joshi A, et al. (2008) Periprosthetic joint infection: The incidence, Timing, and Predisposing Factors. Clinical Orthopaedics and Related Research 466: 1710-1715.

3. Kapadia B, Berg R, Daley J, et al. (2016) Periprosthetic joint infection. The Lancet 387: 386-394.

4. Kirkpatrick C, Alves A, Köhler H, et al. (2000) Biomaterial-induced sarcoma. The American Journal of Pathology, 156: 1455-1467.

5. Montgomery C, Park KJ, Gardner JM, et al. (2019) Post-traumatic sarcomas: Do they exist? 27: 722-728.

6. Bar Y, Merimsky O (2017) Soft-tissue sarcoma following traumatic injury: Case report and review of the literature. Front Oncol 7: 134.

7. Van Mater D, Ano L, Blum JM, et al. (2015) Acute tissue injury activates satellite cells and promotes sarcoma formation via the HGF/c-MET signaling pathway. Cancer Res 75: 605-614.

8. Thomas DM, Ballinger ML (2015) Etiologic, environmental and inherited risk factors in sarcomas. J Surg Oncol 111: 490-495.

9. Visuri T, Pulkkinen P, Paavolainen P (2006) Malignant tumors at the site of total hip prosthesis analytic review of 46 cases. The Journal of Arthroplasty 21: 311-323.

10. S Terrando, A Sambri, Donati D (2017) Angiosarcoma around total hip arthroplasty: Case series and review of the literature. Musculoskelet Surg 102: 21-27.

11. Hughes A, Sherlock D, Hamblen D et al. (1987) Sarcoma at the site of a single hip screw. A case report. The Journal of Bone and Joint Surgery. British 69: 470-472.

12. Delgado ER (1958) Sarcoma following a surgically treated fractured tibia. Clin Orthop 12: 315-318.

13. Yoshida, A Folpe AL (2020) WHO classification of tumours 5th Edition. Soft tissue and bone tumours. International Agency for Research on Cancer. Lyon 3: 122 -123.

14. Dube VE, Fisher DE (1972) Hemangioendothelioma of the leg following metallic fixation. Cancer 30: 1260-1266.

15. Mc Dougall A (1956) Malignant tumor at the site of bone plating. J Bone Joint Surg Br 38: 709-713. 
Citation: Rubio-Saez I, Merino-Rueda LR, Alonso-Sanz J, et al. (2020) Sarcomas Associated with Metal Implants in Orthopedic Surgery and Traumatology. A Report of 3 Cases in 2 Patients. J Orthop Surg Tech 3(2):194-198

16. Himmer O, Lootvoet L, Deprez P, et al. (1991) Angiosarcome apres prothese totale de genou. A propos d'un cas. Rev Chir Orthop Reparatrice Appar Mot 77: 125-128.

17. Weber PC (1986) Epithelioid sarcoma in association with total knee replacement. J Bone Joint Surg 68: 824-826.

18. Junnila M, Pukkala E, Tuomo Visuri, et al. (2014) Cancer incidence and cause-specific mortality in patients with metal-on-metal hip replacements in Finland. Acta Orthopaedica 85: 32-38.

19. Vascellari M, Melchiotti E, Mutinelli F (2006) Fibrosarcoma with typical features of postinjection sarcoma at site of microchip implant in a dog: Histologic and immunohistochemical study. Veterinary Pathology 43: 545-548.

20. Vascellari M, Melchiotti E, Bozza MA, et al. (2003) Fibrosarcomas at presumed sites of injection in dogs: Characteristics and comparison with nonvaccination site fibrosarcomas and feline postvaccinal fibrosarcomas. J Vet Med A Physiol Pathol Clin Med 50: 286-291.

21. Munday JS, Stedman NL, Richey LJ (2003) Histology and immunohistochemistry of seven ferret vaccination-site fibrosarcomas. Vet Pathol 40: 288-293.

22. Carminato A, Vascellari M, Marchioro W, et al. (2011) Micro- chip-associated fibrosarcoma in a cat. Veterinary Dermatology 22: 565-569.

23. Nakamura T, Shimizu Y, Okumura N, et al. (1994) Tumorigenicity of poly-L-lactide (PLLA) plates compared with medical-grade polyethylene. Journal of Biomedical Materials Research 28: 1725.

24. Makela K, Visuri T, Pulkkinen P, et al. (2014) Cancer incidence and cause-specific mortality in patients with metal-on-metal hip replacements in Finland. Acta Orthopaedica 85: 32-38.

25. Radons J (2013) Inflammatory stress and sarcomagenesis: A vicious interplay. Cell Stress and Chaperones 19: 1-13.

26. Lu H, Ouyang $\mathrm{W}$, Huang $\mathrm{C}$ (2006) Inflammation, a key event in cancer development. Mol Cancer Res 4: 221-233.

27. Philip M, Rowley DA, Schreiber H (2004) Inflammation as a tumor promoter in cancer induction. Semin Cancer Biol 14: 433-439.

28. Balkwill F, Mantovani A (2001) Inflammation and cancer: Back to Virchow? Lancet 357: 539-545.

29. Martins Green M, Boudreau N, Bissell MJ (1994) Inflammation is responsible for the development of wound-induced tumors in chickens infected with Rous sarcoma virus. Cancer Res 54: 43344341.

DOI: $10.36959 / 453 / 546$

Copyright: (C) 2020 Israel RS, et al. This is an open-access article distributed under the terms of the Creative Commons Attribution License, which permits unrestricted use, distribution, and reproduction in any medium, provided the original author and source are credited. 